\title{
The benefits and physiological changes of high intensity interval training
}

\author{
Dewi Irawati Soeria Santoso* and Hafizh Ahmad Boenjamin*
}

\begin{tabular}{|c|c|}
\hline ABSTRACT & \\
\hline $\begin{array}{l}\text { Physical inactivity have been linked with many major non-communicable } \\
\text { diseases and as many as } 27.5 \% \text { of adults globally are considered inactive. } \\
\text { Physical activity has been proven to be beneficial in the prevention of } \\
\text { many chronic diseases and may reduce the risk of premature death. High } \\
\text { intensity interval training (HIT) has been gaining popularity as a time- } \\
\text { efficient alternative for regular exercise training. Current studies show that } \\
\text { HIIT is more efficient in improving cardiorespiratory fitness, increasing } \\
\text { insulin sensitivity and reducing blood pressure than moderate intensity } \\
\text { continuous training (MICT). The advantage of HIIT in fat loss compared } \\
\text { to MICT is still unclear, but HIIT might be more efficient in the obese } \\
\text { population. The effect of HIIT on increasing aerobic fitness could be caused } \\
\text { by increase in stroke volume due to the increase in cardiac contractility, } \\
\text { capillary density and mitochondrial adaptation. Fat loss during HIIT could } \\
\text { be caused by increased fat oxidation and elevated hormones that drive }\end{array}$ & $\begin{array}{l}\text { *Department of Medical Physiology, } \\
\text { Faculty of Medicine, } \\
\text { Universitas Indonesia } \\
\text { Correspondence: } \\
\text { Dewi Irawati Soeria Santoso } \\
\text { Department of Medical Physiology, } \\
\text { Faculty of Medicine } \\
\text { Universitas Indonesia } \\
\text { Jl. Salemba Raya No. 6, } \\
\text { Jakarta Pusat, Indonesia } \\
\text { Email: dewi.irawati.1307@gmail.com } \\
\text { Phone: +6281296440098 } \\
\text { ORCID ID: https://orcid.org/0000- } \\
\text { 0001-7390-5298 }\end{array}$ \\
\hline $\begin{array}{l}\text { lipolysis and reduce appetite. While vigorous physical activity may } \\
\text { transiently increase the risk of cardiac events. The effect of HIIT on } \\
\text { increasing aerobic fitness could be caused by increase in stroke volume } \\
\text { due to the increase in cardiac contractility, increased of capillary density } \\
\text { and mitochondrial adaptation. While fat loss during HIIT could be caused } \\
\text { by an increased fat oxidation, elevated hormones that drives lipolysis and } \\
\text { reduces appetite. While vigorous physical activity may transiently increase } \\
\text { the risk of cardiac event. High intensity interval training is generally safe } \\
\text { even in the elderly population and in people with coronary heart disease. }\end{array}$ & $\begin{array}{l}\text { Date of first submission, January } 11 \text {, } \\
2019 \\
\text { Date of final revised submission, } \\
\text { September } 19,2019 \\
\text { Date of acceptance, September } 20 \text {, } \\
2019 \\
\text { This open access article is distributed } \\
\text { under a Creative Commons Attribution- } \\
\text { Non Commercial-Share Alike } 4.0 \\
\text { International License }\end{array}$ \\
\hline $\begin{array}{l}\text { Keywords: High intensity interval training, physiological changes, fat loss, } \\
\text { cardiorespiratory fitness }\end{array}$ & $\begin{array}{l}\text { Cite this article as: Santoso DIS, } \\
\text { Boenjamin HA. The benefits and } \\
\text { physiological changes of high intensity } \\
\text { interval training. Univ Med 2019;38: } \\
\text { 209-16. doi: } 10.18051 / \text { UnivMed.2019. } \\
\text { v38.209-216 }\end{array}$ \\
\hline
\end{tabular}




\section{INTRODUCTION}

Physical inactivity is now the fourth highest risk factor in global mortality. ${ }^{(1)}$ It is associated with many non-communicable diseases such as obesity, hypertension, cardiovascular diseases, type 2 diabetes and cancer. ${ }^{(2)}$ Inactivity is thought to be responsible for $9 \%$ of premature mortality worldwide in 2008. ${ }^{(3)}$ Physical activity has been proven to be beneficial in the prevention of many chronic diseases and may reduce the risk of premature death. ${ }^{(4)}$ It is recommended by the World Health Organization and U.S. Department of Health and Human Services that adults should perform 150-300 minutes of moderate intensity or 75-150 minutes of vigorous intensity aerobic physical activity per week. ${ }^{(1,5)}$ However, a recent study has found that globally as many as $27.5 \%$ of adults are considered inactive, ${ }^{(6)}$ and one of the main perceived barriers to exercise is the lack of time. ${ }^{(7)}$

To solve this problem, one of the exercise options that has been gaining popularity is the practice of high intensity interval training (HIIT). ${ }^{(8)}$ High intensity interval training is considered to be more time-efficient and effective than moderate intensity continuous training (MICT). Here we will examine the benefits of HIIT and the current supporting evidence.

\section{High intensity interval training}

High intensity interval training is a form of exercise in which there is a short burst of vigorous activity followed by a period of recovery by rest or low-intensity exercise before starting another bout. ${ }^{(9)}$ Depending on the presence of a lactate steady state, HIIT can be categorized as aerobic or anaerobic. ${ }^{(10)}$

The high intensity during HIT is completed above the anaerobic threshold. However recently researchers have also begun differentiating the varying level and duration of the high intensity in HIIT with sprint or supramaximal-intensity interval training (SIT). The intervals in HIIT last 1-4 minutes above the anaerobic threshold and up to $\mathrm{VO}_{2}$ max, while SIT intervals last 20-30 seconds above $150 \% \mathrm{VO}_{2}$ max power. ${ }^{(11)}$ The target intensity in HIIT is usually measured by the heart rate; often the target is based on the heart rate reserve (HRR) or the maximum heart rate (MRR). ${ }^{(12)}$ The interval nature of HIIT allows a period of recovery so that multiple rounds of high intensity activity can be performed. There is no main or official protocol for HIIT, but some of the more popular protocols of HIIT/SIT summarized by Cress et al. ${ }^{(12)}$ are listed in Table 1.

High intensity interval training can be done with various modalities and equipment, and often cycle ergometers are used in this activity. The benefit of using a cycle ergometer is that the total amount of work and power can be measured. Running tracks, swimming pools, arm ergometers, body weight and resistance training are also commonly used for this activity. (12) It is recommended to measure the heart rate to make sure that the targeted intensity is achieved.

One of the benefits of HIIT compared to other activities is its time efficiency, the whole activity of HIIT can be done in a relatively short time compared to traditional forms of exercise. So HIIT is considered an attractive exercise modality for people with a busy lifestyle.

\section{Benefits of HIIT}

There are a lot of studies on the beneficial effects of HIIT. One of the most common targeted outcomes of HIIT is fat loss. While there are many protocols for HIIT, a study has shown that both short and long periods of high intensity exercise during HIIT have resulted in a reduction in fat mass, fat percentage as well as an increase in physical endurance (VO2max) with no significant differences between the two groups. ${ }^{(13)}$ Studies have also compared the amount of fat loss between HIIT and continuous exercise. While it is suggested that HIIT is more efficient in causing fat loss, a meta-analysis by Keating et al., ${ }^{(14)}$ who analyzed 31 studies that compared the effect of fat loss by HIIT / SIT and MICT, showed no significant difference in the amount of reduced total body fat and fat mass when similar time 
Table 1. Popular HIIT / SIT protocols ${ }^{(12)}$

\begin{tabular}{|c|c|c|c|c|c|}
\hline Name & $\begin{array}{l}\text { Work Interval } \\
\text { and Intensity }\end{array}$ & $\begin{array}{l}\text { Rest Interval } \\
\text { and Intensity }\end{array}$ & Series & Modality & $\begin{array}{l}\text { Total Time } \\
\text { (Including } \\
\text { Warm-up and } \\
\text { Cooldown) }\end{array}$ \\
\hline Tabata & $\begin{array}{l}20 \text { Seconds } \\
170 \% \mathrm{VO}_{2} \max \end{array}$ & $\begin{array}{l}10 \text { seconds } \\
\text { Rest or very } \\
\text { low intensity }\end{array}$ & 8 times & $\begin{array}{l}\text { Cycle ergometer, track, } \\
\text { treadmill, body weight or } \\
\text { resistance exercises }\end{array}$ & 4 minutes \\
\hline Wingate & $\begin{array}{l}30 \text { seconds all } \\
\text { out against } \\
\text { constant force }\end{array}$ & $\begin{array}{l}4 \text { minutes } \\
\text { Active } \\
\text { recovery low } \\
\text { intensity }\end{array}$ & 4-6 times & $\begin{array}{l}\text { Mechanically braked } \\
\text { cycle ergometer }\end{array}$ & $18-27$ minutes \\
\hline Conventional & $\begin{array}{l}60 \text { seconds } \\
>90 \% \text { HRR }\end{array}$ & $\begin{array}{l}60 \text { seconds } \\
\text { Rest or active } \\
\text { recovery }\end{array}$ & 10 times & $\begin{array}{l}\text { Cycle ergometer, track, } \\
\text { treadmill, body weight or } \\
\text { resistance exercises }\end{array}$ & 20 minutes \\
\hline Clinical & $\begin{array}{l}4 \text { minutes } \\
85 \%-95 \% \mathrm{MHR}\end{array}$ & $\begin{array}{l}3 \text { minutes } \\
60 \%-70 \% \\
\text { MHR }\end{array}$ & 4 times & $\begin{array}{l}\text { Treadmill with handrails, } \\
\text { cycle ergometer, arm } \\
\text { ergometer }\end{array}$ & 25 minutes \\
\hline
\end{tabular}

commitment and energy is used. However, a different meta-analysis that examined 13 studies about the effect of HIIT on an obese population showed that HIIT produces similar effects of fat loss compared to MICT, even when the time committed for HIIT in this study was $40 \%$ less (shorter) than that in the MICT group. ${ }^{(15)}$ One study on obese young women found that there was a comparable decrease of abdominal visceral fat in the HIIT group and the MICT group after 12 weeks of exercise even when the duration of the MICT sessions was almost double that of the HIIT sessions. ${ }^{(16)}$ This result may show that when the targeted outcome is the amount of fat loss, HIIT is more efficient in the obese population, but in the general population it has the same effectiveness as MICT.

A targeted outcome for which HIIT might be more beneficial than MICT is cardiorespiratory fitness. A meta-analysis that compared the increase in $\mathrm{VO}_{2}$ max, as an indicator for increased aerobic fitness, between endurance training and HIIT showed a greater $\mathrm{VO}_{2}$ max gain in the HIIT group. ${ }^{(17)}$ Another meta-analysis in a special population with coronary artery disease also showed that HIIT is more beneficial in increasing cardiorespiratory fitness compared to MICT. ${ }^{(18)}$ Even a study on the elderly population, with a mean age of over 60 , showed a positive effect of HIIT on their overall cardiorespiratory fitness. ${ }^{(19)}$
With different protocols, a meta-analysis showed that long interval, high volume and moderate to long-term HIIT gave the greatest benefit in improving $\mathrm{VO}_{2}$ max. ${ }^{(20)}$

Another documented effect of HIIT is the reduction in blood pressure. One meta-analysis that compared the effect of HIIT and MICT on resting blood pressure in individuals with prehypertension and hypertension, showed that both groups had a positive and similar effect on the reduction of both resting systolic and diastolic blood pressure. (21) However, in some of those studies in the meta-analysis, the exercise time was less in the HIIT than in the MICT groups. Another meta-analysis of 16 studies on the 24hour blood pressure reduction effect between HIIT and MICT showed that HIIT had a greater decrease of night-time diastolic blood pressure compared to MICT. ${ }^{(22)}$ Individuals with type 2 diabetes also seem to benefit more from HIIT. A meta-analysis showed that compared to continuous training, HIIT was more effective in reducing insulin resistance and showed significant reductions in $\mathrm{HbAlc} .^{(23)}$

One of the advantages of HIIT is that it needs less time than conventional continuous training. Therefore it is hoped that more people will adhere to the programs, but the results of the studies on adherence have been mixed. One meta-analysis examined the acute affective and 
enjoyment response of HIIT and MICT on different scales of interest. High intensity interval training had beneficial effects in most of the studies but showed similar or identical results on some scales of interest, and small and trivial increases on other scales compared to MICT. ${ }^{(24)}$ Several short-term studies (4 to 8 weeks) have shown that compared to MICT, participants in HIIT expressed greater enjoyment and adherence for the program. ${ }^{(25,26)}$ One study that combined both supervised and independent rounds of HIIT compared to MICT for 8 weeks in obese adults, showed no differences between the level of enjoyment and adherence of HIIT compared to MICT. ${ }^{(27)}$ One long-term study on the adherence of unsupervised HIIT in an obese population for 12 months showed that there was a significant decrease in adherence from $60.8 \%$ at baseline to $19.6 \%$ at the end of the study. ${ }^{(28)} \mathrm{In}$ different age groups, studies on young adults in one university have also shown low adherence to the programs. ${ }^{(29)}$ Meanwhile a study on the aging population have shown good adherence to the program. ${ }^{(19)}$ One of the possible biases in these studies might come from the nature of the supervision during the research. The level of intensity may also affect the level of enjoyment; HIIT performed at higher intensity with low recovery time ratio may bring a negative effect on the overall enjoyment of HIIT. ${ }^{(30)}$ To effectively know the real adherence rate of HIIT, more long-term unsupervised studies on different populations have to be conducted.

\section{Physiological changes in HIIT}

Several acute physiological responses that were observed after a session of HIIT include increase in heart rate, cortisol, catecholamines, growth hormone, glucose and plasma lactate levels, glycerol, depletion of adenosine triphosphate (ATP), phosphocreatine ( $\mathrm{PCr}$ ) and glycogen stores as well as a decrease in parasympathetic reaction. ${ }^{(31)}$ The increase in heart rate may differ depending on the HIIT protocol used, however higher peak heart rate is observed in long exercises compared to the short ones. ${ }^{(32)}$ Hormones such as epinephrine, norepinephrine, growth hormone, interleukin 6 , free cortisol and adrenocorticotropic hormone $(\mathrm{ACTH})$ are all increased after HIIT. ${ }^{(33)}$ Compared to moderate intensity exercise, the increases in adrenocorticotropic hormone $(\mathrm{ACTH})$, free cortisol and growth hormone were higher after HIIT. ${ }^{(33)}$ This may be significant, as catecholamine, cortisol and growth hormone play a role in lipolysis. ${ }^{(34)}$

The lactate level also increases during HIIT and the increase seems to be higher in trained athletes. ${ }^{(35)}$ Another study that compared HIIT to high volume training (HVT), also found that 5 weeks of HIIT intervention significantly increase maximal lactate concentration while HVT decreases it. A higher rate of lactate accumulation correlates with higher anaerobic energy yield, which could lead to better performance. ${ }^{(36)}$ HIIT also seems to increase the release of fatty acids, as indicated by the increased levels of glycerol. ${ }^{(35)}$ Blood glucose is increased after HIIT and is higher than after moderate intensity exercise. ${ }^{(33)}$ Parasympathetic activation also seems to decrease after repeated sprint exercise; this might be caused by the increase in sympathetic activity as well as the continued elevation of adrenergic factors and other local metabolites (epinephrine, norepinephrine, $\mathrm{H}^{+}$, lactate, etc.) during recovery. ${ }^{(37)}$ In the metabolic response, first ATP and PCr were decreased in HIIT followed with a decrease in glycogen stores by anaerobic glycolysis. ${ }^{(31,38,39)}$

The effect of HIIT in increasing cardiorespiratory fitness may be caused by multiple factors. The beneficial effect for aerobic fitness by HIIT is likely to be caused by central and peripheral adaptation. ${ }^{(40)}$ Central adaptation includes increase in stroke volume caused by the increase in cardiac contractility. ${ }^{(31,41)}$ Peripheral adaptations include increased capillary density and mitochondrial adaptation. ${ }^{(40)}$ A study in skeletal muscle adaptation showed an improvement in mitochondrial content and activity. ${ }^{(40)}$ Another contribution to aerobic fitness might be the result of the degradation of 
phosphocreatine during repeated HIIT. ${ }^{(31)}$ In anaerobic fitness, a study showed an increase in anaerobic capacity after two weeks of SIT. ${ }^{(42)}$

The theorized mechanism for fat loss during HIIT includes an increased in high intensity exercise, fat oxidation and the loss of appetite. ${ }^{(31)}$ Repeated high intensity exercise may cause an inhibition of anaerobic glycogenolysis, so that ATP is derived mainly from intramuscular triacylglycerol storage and $\mathrm{PCr}$ degradation. ${ }^{(43)}$ Increased venous glycerol after HIIT could be caused by the release of fatty acids from adipose and intramuscular triacylglycerol stores. ${ }^{(35)}$ Other studies have also shown that after a number of sessions in HIIT there was an increased capacity for the oxidation of fatty acids in skeletal muscle and the whole body. ${ }^{(44)}$ Elevated hormones such as growth hormone, catecholamine and cortisol may also play a role in driving lipolysis. ${ }^{(33,34)} \mathrm{In}$ rats, hard exercise has been repeatedly reported to reduce food intake and reduce food intake by facilitating the release of corticotropin releasing factor (CRF) a potent anorectic peptid. ${ }^{(45)}$ Animal studies in rats have also shown that high intensity activities such as running facilitate the release of corticotrophin releasing factor, a potent anorectic peptide. ${ }^{(45)}$ A recent study on individuals with type 2 diabetes has also found that HIIT influences appetite after 12 weeks by stimulating appetite hormones, reducing TNF- $\alpha$, peptide YY (PYY) and ghrelin and increasing glucagon-like peptide1 (GLP-1). However, the changes in appetite hormones may differ between lean and obese participants, with obese patients with type 2 diabetes being more likely to elicit appetite hormones in HIIT. ${ }^{(46)}$

The mechanism of insulin sensitivity in HIIT is still unclear. Increased insulin sensitivity might be caused by successful weight loss. (47) However, this may not be the only factor that leads to a decrease in insulin resistance. One other possible mechanism is through skeletal muscle adaptation, which can increase its fatty acid oxidation capacity and glycolytic enzymes. ${ }^{(31)}$ Increased muscle glucose uptake may be mediated by a high degree of muscle fiber recruitment and use of glycogen. ${ }^{(48)}$ The increase in insulin-independent glucose transporter type 4 (GLUT4) translocation or muscle GLUT4 content may also play a part. ${ }^{(48)}$

The blood pressure reduction in HIT may come from the improvement in endothelial function. One study has shown that HIIT in older hypertensive patients increased apelin and nitric oxide, a factor in endothelial vasodilatation, and decreased endothelin-1.(49) Better vascular function outcome in HIIT may also be caused by increased shear stress in the arterial wall. ${ }^{(22)}$

\section{Adverse effects}

The high intensity in HIIT has been considered for adverse effects, since vigorous physical activity may transiently increase the risk of cardiovascular events. ${ }^{(50)}$ However, current evidence reveals that HIIT is relatively safe, even in individuals with a cardiac condition. A metaanalysis that compared the effect of HIIT and MICT in cardiac rehabilitation showed that there were no recorded events of death or cardiac events that required hospitalization in 953 participants (465 HIIT and 488 MICT). ${ }^{(18)}$ Another study that examined the use of MICT and HIIT in 4846 coronary heart disease patients, showed that the risk of any cardiovascular event was low in both groups, with only a single fatal incident reported in the MICT group after over 129,456 exercise hours and two non-fatal incidents in the HIIT group after over 46,364 exercise hours. ${ }^{(51)}$

\section{CONCLUSION}

When compared to MICT, HIIT is superior in improving cardiorespiratory fitness, reducing blood pressure and increasing insulin sensitivity. While some have been cautious about the nature of the high intensity, HIIT is generally safe even in special populations, such as elderly people or individuals with coronary heart disease. The advantage of adherence in HIIT compared to MICT is not clear, since some studies have shown greater enjoyment and adherence levels but 
others have shown similar levels in MICT. These results suggest that choosing HIIT should be based on personal preference. Current limitations in HIIT research are the lack of uniformity between protocols used in the research and lack of long-term research.

\section{CONFLICT OF INTEREST}

There is no potential conflict of interest and financial support to disclose.

\section{CONTRIBUTORS}

DISS and HAB conceived of the review, identified and interpreted relevant studies for inclusion, wrote the manuscript, and critically revised the manuscript. Both authors have read and approved the final manuscript and agreed to be accountable for all aspects of the work.

\section{REFERENCES}

1. World Health Organization. Global recommendations on physical activity for health. Geneva: World Health Organization; 2010.

2. Booth F, Roberts C, Laye M. Lack of exercise is a major cause of chronic diseases. Compr Physiol 2012;2:1143-211. doi: 10.1002/cphy.c110025.

3. Lee I, Shiroma E, Lobelo F, et al. Effect of physical inactivity on major non-communicable diseases worldwide: an analysis of burden of disease and life expectancy. Lancet 2012;380:219-29. doi: 10.1016/S0140-6736(12)61031-9.

4. Warburton D. Health benefits of physical activity: the evidence. CMAJ 2006;174:801-9. doi: 10.1503/ cmaj.051351.

5. Piercy KL, Troiano RP, Ballard RM, et al. The physical activity guidelines for Americans. JAMA 2018; 320:2020. doi:10.1001/jama.2018.14854.

6. Guthold R, Stevens G, Riley L, et al. Worldwide trends in insufficient physical activity from 2001 to 2016: a pooled analysis of 358 population-based surveys with 1.9 million participants. Lancet Glob Health 2018;6:e1077-e86. doi: 10.1016/s2214109x(18)30357-7.

7. Hoare E, Stavreski B, Jennings G, et al. Exploring motivation and barriers to physical activity among active and inactive Australian adults. Sports 2017;5:47. doi: 10.3390/sports5030047.
8. Thompson WR. Worldwide survey of fitness trends for 2018. ACSM'S Health Fit J 2017;21:10 9. doi: 10.1249/FIT.0000000000000341.

9. Feito Y, Heinrich K, Butcher S, et al. High intensity functional training (HIFT): definition and research implications for improved fitness. Sports 2018;6:76. doi: 10.3390/sports6030076.

10. Cipryan L, Tschakert G, Hofmann P. Acute and post-exercise physiological responses to highintensity interval training in endurance and sprint athletes. J Sports Sci Med 2017;16:219-29.

11. Talanian JL. Defining different types of interval training: Do we need to use more specific terminology? Sport Exerc Med Open J 2015;1:1613. doi: 10.17140/SEMOJ-1-124.

12. Cress M, Porcari J, Foster C. Interval training. ACSMs Health Fit J 2015;19:3-6.

13. Alves E, Salermo G, Panissa V, et al. Effects of long or short duration stimulus during highintensity interval training on physical performance, energy intake, and body composition. J Exerc Rehabil 2017;13:393-9. doi: 10.12965/jer.1734962.481.

14. Keating S, Johnson N, Mielke G, et al. A systematic review and meta-analysis of interval training versus moderate-intensity continuous training on body adiposity. Obes Rev 2017;18:943-64. doi: 10.1111/obr.12536.

15. Wewege M, van den Berg R, Ward R, et al. The effects of high-intensity interval training vs. moderate-intensity continuous training on body composition in overweight and obese adults: a systematic review and meta-analysis. Obes Rev 2017;18:635-46. doi: 10.1111/obr.12532.

16. Zhang H, Tong T, Qiu W, et al. Comparable effects of high-intensity interval training and prolonged continuous exercise training on abdominal visceral fat reduction in obese young women. $\mathrm{J}$ Diabetes Res 2017;2017:1-9. doi: 10.1155/2017/ 5071740.

17. Milanović Z, Sporiš G, Weston M. Effectiveness of high-intensity interval training (HIT) and continuous endurance training for $\mathrm{VO} 2 \mathrm{max}$ improvements: a systematic review and metaanalysis of controlled trials. Sports Med 2015;45:1469-81. doi: 10.1007/s40279-015-0365-0.

18. Hannan A, Hing W, Simas V, et al. High-intensity interval training versus moderate-intensity continuous training within cardiac rehabilitation: a systematic review and meta-analysis. J Sports Med 2018;9:1-17. doi: 10.2147/OAJSM.S150596.

19. Knowles A, Herbert P, Easton C, et al. Impact of low-volume, high-intensity interval training on maximal aerobic capacity, health-related quality of life and motivation to exercise in ageing men. 
Age (Dordr) 2015;37:25. doi: 10.1007/s11357-0159763-3.

20. Wen D, Utesch T, Wu J, et al. Effects of different protocols of high intensity interval training for VO2max improvements in adults: a meta-analysis of randomised controlled trials. J Sci Med Sport 2019;22:941-7. doi: 10.1016/j.jsams.2019.01.013.

21. Costa E, Hay J, Kehler D, et al. Effects of highintensity interval training versus moderateintensity continuous training on blood pressure in adults with pre- to established hypertension: a systematic review and meta-analysis of randomized trials. Sports Med 2018;48:2127-42. doi: 10.1007/s40279-018-0944-y.

22. Way K, Sultana R, Sabag A, et al. The effect of high intensity interval training versus moderate intensity continuous training on arterial stiffness and $24 \mathrm{~h}$ blood pressure responses: a systematic review and meta-analysis. J Sci Med Sport 2018; 22:385-91. doi: 10.1016/j.jsams.2018.09.228.

23. Jelleyman C, Yates T, O'Donovan G, et al. The effects of high-intensity interval training on glucose regulation and insulin resistance: a metaanalysis. Obes Rev 2015;16:942-61. doi: 10.1111/ obr.12317.

24. Oliveira B, Santos T, Kilpatrick M, et al. Affective and enjoyment responses in high intensity interval training and continuous training: a systematic review and meta-analysis. PLoS ONE 2018;13:e0197124. doi: 10.1371/journal.pone. 0197124.

25. Heinrich K, Patel P, O'Neal J, et al. High-intensity compared to moderate-intensity training for exercise initiation, enjoyment, adherence, and intentions: an intervention study. BMC Public Health 2014;14:789. doi: 10.1186/1471-2458-14-789.

26. Jung M, Bourne J, Beauchamp M, et al. Highintensity interval training as an efficacious alternative to moderate-intensity continuous training for adults with prediabetes. J Diabetes Res 2015.Article ID 191595, 9 pages. DOI: http:// dx.doi.org/10.1155/2015/191595.

27. Vella C, Taylor K, Drummer D. High-intensity interval and moderate-intensity continuous training elicit similar enjoyment and adherence levels in overweight and obese adults. Eur J Sport Sci2017;17:1203-11. doi: 10.1080/17461391.2017. 1359679.

28. Roy M, Williams S, Brown R, et al. High-intensity interval training in the real world. Med Sci Sports Exerc 2018;50:1818-26. doi: 10.1249/MSS. 0000000000001642.

29. Eather N, Riley N, Miller A, et al. Efficacy and feasibility of HIIT training for university students: the Uni-HIIT RCT. J Sci Med Sport 2018; 22:59660. doi: 10.1016/j.jsams.2018.11.016.
30. Oliveira B, Slama F, Deslandes A, et al. Continuous and high-intensity interval training: which promotes higher pleasure? PLoS ONE 2013;8: e79965. doi: 10.1371/journal.pone.0079965.

31. Boutcher S. High-intensity intermittent exercise and fat loss. J Obes 2011; Article ID 868305, 10 pages. DOI: http://dx.doi.org/10.1155/2011/ 868305.

32. Warr-di Piero D, Valverde-Esteve T, RedondoCastán J, et al. Effects of work-interval duration and sport specificity on blood lactate concentration, heart rate and perceptual responses during high intensity interval training. PLoS ONE2018;13:e0200690. doi: 10.1371/journal. pone. 0200690 .

33. Peake J, Tan S, Markworth J, et al. Metabolic and hormonal responses to isoenergetic highintensity interval exercise and continuous moderate-intensity exercise. Am J Physiol Endocrinol Metab 2014;307:E539-E52. doi: 10.1152/ajpendo.00276.2014.

34. Nielsen T, Jessen N, Jørgensen J, et al. Dissecting adipose tissue lipolysis: molecular regulation and implications for metabolic disease. J Mol Endocrinol 2014;52:R199-R222. doi: 10.1530/JME13-0277.

35. Trapp E, Chisholm D, Boutcher S. Metabolic response of trained and untrained women during high-intensity intermittent cycle exercise. Am J Physiol Regul Integr Comp Physiol 2007;293: R2370-R5. doi: 10.1152/ajpregu.00780.2006.

36. Sperlich B, Zinner C, Heilemann I, et al. Highintensity interval training improves VO2peak, maximal lactate accumulation, time trial and competition performance in 9-11-year-old swimmers. Eur J Appl Physiol 2010;110:1029-36. doi: 10.1007/s00421-010-1586-4.

37. Buchheit $\mathrm{M}$, Laursen $\mathrm{P}$, Ahmaidi S. Parasympathetic reactivation after repeated sprint exercise. Am J Physiol Heart Circ Physiol 2007;293:H133-H41. doi: 10.1152/ajpheart.00062. 2007.

38. Burgomaster K, Heigenhauser G, Gibala M. Effect of short-term sprint interval training on human skeletal muscle carbohydrate metabolism during exercise and time-trial performance. J Appl Physiol 2006;100:2041-7. doi: 10.1152/jappl physiol.01220.2005.

39. Tomlin D, Wenger $\mathrm{H}$. The relationship between aerobic fitness and recovery from high intensity intermittent exercise. Sports Med 2001;31:1-11. doi: 10.2165/00007256-200131010-00001.

40. MacInnis M, Gibala M. Physiological adaptations to interval training and the role of exercise intensity. J Physiol 2016;595:2915-30. doi: 10.1113/ JP273196. 
41. Helgerud J, Høydal K, Wang E, et al. Aerobic highintensity intervals improve VO2max more than moderate training. Med Sci Sports Exerc 2007;39: 665-71. doi: 10.1249/mss.0b013e3180304570.

42. Whyte L, Gill J, Cathcart A. Effect of 2 weeks of sprint interval training on health-related outcomes in sedentary overweight/obese men. Metabolism 2010;59:1421-8. doi: 10.1016/j.metabol.2010.01.002.

43. Hearris MA, Hammond KM, Fell JM, et al. Regulation of muscle glycogen metabolism during exercise: implications for endurance performance and training adaptations. Nutrients 2018;10: 298. doi:10.3390/nu10030298.

44. Talanian J, Galloway S, Heigenhauser G, et al. Two weeks of high-intensity aerobic interval training increases the capacity for fat oxidation during exercise in women. J Appl Physiol 2007; 102:143947. doi: 10.1152/japplphysiol.01098.2006.

45. Bilski J, Teległow A, Zahradnik-Bilska J, et al. Effects of exercise on appetite and food intake regulation. Med Sport 2009;13:82-94. doi: 10.2478/ v10036-009-0014-5.

46. Afrasyabi S, Marandi S, Kargarfard M. The effects of high intensity interval training on appetite management in individuals with type 2 diabetes: influenced by participants weight. J Diab Metab Dis 2019;18:107-17. doi: 10.1007/ s40200-019-00396-0.
47. Clamp L, Hume D, Lambert E, et al. Enhanced insulin sensitivity in successful, long-term weight loss maintainers compared with matched controls with no weight loss history. Nutrition Diabetes 2017;7:e282.doi: 10.1038/nutd.2017.31.

48. Gillen J, Little J, Punthakee Z, et al. Acute highintensity interval exercise reduces the postprandial glucose response and prevalence of hyperglycaemia in patients with type 2 diabetes. Diabetes Obes Metab 2012;14:575-7. doi: 10.1111/j.1463-1326.2012.01564.x.

49. Izadi M, Ghardashi AA, Asvadi FM, et al. Highintensity interval training lowers blood pressure and improves apelin and NOx plasma levels in older treated hypertensive individuals. J Physiol Biochem 2017;74:47-55. doi: 10.1007/s13105-0170602-0.

50. Franklin B, Billecke S. Putting the benefits and risks of aerobic exercise in perspective. Current Sports Medicine Reports 2012;11:201-8. doi: 10.1249/JSR.0b013e31825dabd4.

51. Rognmo O, Moholdt $\mathrm{T}$, Bakken $\mathrm{H}$, et al Cardiovascular risk of high- versus moderateintensity aerobic exercise in coronary heart disease patients. Circulation 2012;126:1436-40. doi: 10.1161/CIRCULATIONAHA.112.123117. 